\title{
Molecular Dynamics Simulations of Plasticity and Crackingin Lithiated Silicon Electrodes
}

\author{
Haoran Wang, Huck Beng Chew* \\ Department of Aerospace Engineering, University of Illinois at Urbana-Champaign, Urbana, IL \\ 61801, USA \\ *Corresponding Author. Email: hbchew@illinois.edu
}

\begin{abstract}
First-principle calculations have provided critical insights into thedeformationbehavior of lithiated silicon electrodes in high-capacity lithium ion batteries, but quantitative interpretations have been limited by the size scales of these calculations. Here, we show that large-scale molecular dynamics (MD) simulations, based on the modified embedded atom method (MEAM) potential, are capable of describing the elastic softening and plastic behavior of $\mathrm{Li}_{x} \mathrm{Si}$ alloys. In particular, our MD simulations at $0 \mathrm{~K}$ correctly reproduce the stress-strain response of $\mathrm{Li}_{x} \mathrm{Si}$ alloys from Density Functional Theory (DFT) calculations across all Li concentrations, while matching the corresponding yield strength data from existing experiments at $300 \mathrm{~K}$. Results from these MD simulations reveala sharp transition in the atomic-scale plasticity mechanisms for $\mathrm{Li}_{x} \mathrm{Si}$ with increasing Li content: from the breaking of Li-Si bonds at $x<1$, to the breaking of Li-Li bonds at $x>1$. The associated transition in the fracture behavior from brittletoductile isdue to the high stretchability of Li-Li bonds compared to Li-Si and Si-Si bonds.
\end{abstract}

Keywords: silicon electrode; plasticity; failure; molecular dynamics; interatomic potential

\section{Introduction}

Lithium ion batteries store energy by inserting lithium ions into solid electrodes. Conventional lithium ion batteriesuse graphite electrodes, which store a single Li atom per $6 \mathrm{C}$ atoms. In comparison, Si electrodes have order-of-magnitude higher specific capacities, since each Si atom can theoretically bond with $\sim 3.75 \mathrm{Li}$ atoms[1]. However, this enormous storage of $\mathrm{Li}$ atoms in the Si electrode leads to unprecedented volume expansion of $\sim 300 \%$ when the 
electrode is fully-lithiated[2]. The huge inhomogeneous volume changes during cycles of lithiation and delithiation cause massive cracking of the Si electrode, eventually resulting in the loss of electrical contact and consequent capacity fade[3-5].There is now increasing evidence that the mechanical degradation of Si electrodes under electrochemical cycling can be mitigated through the use of electrodes of small feature sizes, such as nanowires, nanoparticles, porous configurations, thin films, and core-shell structures[6-11]. Such nanostructured Si electrodes display significantly higher reversible charge capacity and longer cycle life, because the small characteristic dimensions of these electrodes lead to reduced energy release rate which is insufficient to drive crack propagation. More intriguingly, it is observed that these lithiated $\mathrm{Si}$ electrodes also undergo plastic deformation[12-15], which is in contrast to the brittle nature of unlithiated $\mathrm{Si}$; how this plasticity behavior of $\mathrm{Li}_{x}$ Siinfluences thefracture propertiesis also contradictory. Early experiments have reported that both unlithiated and lithiated Si electrodes share similar fracture toughness values[16]. More recent experiments and computations concluded that the lithiation-induced plasticity of $\mathrm{Li}_{x} \mathrm{Si}$ alloys results in ultra-high flaw tolerance[17,18].

A variety of continuum theories have been introduced to describe the coupled Li diffusion and elasto-plasticity behaviorof $\mathrm{Li}_{x} \mathrm{Si}$ alloys[19,20].These models commonlyaccount forfinite deformation,as well as stress-gradient effects on Li diffusion. More recent models incorporate the contributionsfrom lithiation kinetics and anisotropic motion of phase boundariesto better describe the lithiation of crystalline $\mathrm{Si}[21]$.However, the physical properties used in these continuum models, including the elastic modulus and plastic yield strength of $\mathrm{Li}_{x} \mathrm{Si}$ as a function of lithium contentx, have to be calibrated from non-trivial experimental measurements or firstprinciple calculations[18,22-24].More importantly, the underlying mechanisms of lithiationinduced deformation are inherently atomistic in nature. For example, the insertion of $\mathrm{Li}$ atoms into Si results in the breaking of covalent Si-Si bonds and the forming of ionic Si-Li bonds; such significant changes to the chemical bonding cannot be described by continuum models.

Density functional theory (DFT) calculations have been used to characterize the elastoplastic behavior of amorphous $\mathrm{Li}_{x} \mathrm{Si}$ alloys. Shenoy et al. demonstrated the elastic softening of $\mathrm{Li}_{x} \mathrm{Si}$ alloys with increasing $\mathrm{Li}$ concentration[23]. Wang et al. showed the decreasing yield strength of $\mathrm{Li}_{x} \mathrm{Si}$ with increasing $\mathrm{Li}$ content, and reported a sharp transition in the failure 
response - from brittle to ductile - at intermediate Li concentrations[18]. These first-principle studies fully account for the bonding nature of $\mathrm{Li}_{x} \mathrm{Si}$ under deformation. However, interpretations of the plasticity and fracture processes have been limited by the simulation domain sizes of 150 atoms. In addition, temperature effects are not considered in these quantum-mechanical calculations. Accordingly, the DFT calculations are reported to over predict the yield strengths of $\mathrm{Li}_{x} \mathrm{Si}$ compared to experimental measurements[22,25].

Molecular dynamics (MD) simulations offer an intermediate approach to link the DFT calculations to experiments. Two classes of interatomic potentials have so far been developed to describe the deformation behavior of $\mathrm{Li}_{x} \mathrm{Si}$ alloys: the reactive-force-field (ReaxFF) potential by Ostadhosseinet al.[26]and the modified embedded atomic method (MEAM) by Cui et al.[27] In this paper, we compare the MD stress-strain predictions of $\mathrm{Li}_{x} \mathrm{Si}$ alloys using both the ReaxFF and MEAM potentials. Results from the latter at $0 \mathrm{~K}$ are found to reproduce the elastic modulus and yield strength data of $\mathrm{Li}_{x} \mathrm{Si}$ across all $x$ from DFT calculations, while correctly matching the experimental measurements at $300 \mathrm{~K}$. Our MEAM-based MD simulations further demonstrate that a critical simulation domain size of $\sim 15 \mathrm{~nm}$ is necessary to capture the micromechanics of cleavage fracture at lower Li concentrations $(x<1)$ and the delocalized failure response at higher Li concentrations $(x \geq 1)$. This transition in the fracture behavior from brittle to ductile corresponds with a transition in the atomic-scale plasticity mechanism, from the breaking of stiffer Li-Si bonds at low Li concentrations, to the breaking of highly stretchable Li-Li bonds at high Li concentrations.

\section{Molecular Dynamics Modeling}

Our MD simulationsare performed using the Large-scale Atomic/Molecular Massively Parallel Simulator (LAMMPS)[28].The interatomic interactions are governed by MEAM and ReaxFF interatomic potentials specifically developed to describe the deformation behavior of pure $\mathrm{Si}$ and/orLi ${ }_{x} \mathrm{Si}$ alloys[26,27,29].We create amorphousSi, $\mathrm{LiSi}_{2}, \mathrm{LiSi}, \mathrm{Li}_{12} \mathrm{Si}_{7}$ and $\mathrm{Li}_{15} \mathrm{Si}_{4}$ model structures via a rapid heating and quenching method first introduced in our previous DFT studies[18].We start with realistic initial model structures,obtained by replicating and truncating the final periodic atomic $\mathrm{Li}_{x} \mathrm{Si}$ configurations obtained from DFT[18],to achievesixfully-periodic cubic simulation boxeswith initial edge dimensions of $\mathrm{d}=2.5,4,7,10,12$, and $15 \mathrm{~nm}$ for each 
$\mathrm{Li}_{x} \mathrm{Si}$ alloy.We perform an NVT calculation by subjecting each model structure to a temperature of $3000 \mathrm{~K}$ while keeping the box dimensions fixed; the temperature is maintained by a Berendson thermostat for 10,000 MD steps. This temperature is higher than the melting point of $\mathrm{Li}, \mathrm{Si}$, and their compounds, and allows for sufficient intermixing between the $\mathrm{Li}$ and $\mathrm{Si}$ atoms.Each amorphous model structure is then quenched to the target temperature of $300 \mathrm{~K}$ (or 0 K) within 20,000 MD steps. We then perform an NPT calculation by equilibrating the structure at $300 \mathrm{~K}$ (or $0 \mathrm{~K}$ ) for a further 30,000 MD steps while allowing the simulation box to relax with a Berendson barostat to achieve a stress-free configuration. The final relaxed atomic configurations for a $\sim 4 \times 4 \times 4 \mathrm{~nm}^{3} \mathrm{MD}$ simulation box, based on the MEAM and ReaxFF interatomic potentials, are shown in Fig. 1. In all our simulations, we use a fixed time step of $1 \mathrm{fs}$.

We perform a pair correlation analysis to quantify the bonding characteristicsfor the $\mathrm{Si}-\mathrm{Si}$, $\mathrm{Li}-\mathrm{Si}$ and $\mathrm{Li}$-Li bonds within each $\mathrm{Li}_{x} \mathrm{Si}$ model structure in Fig. 1. We show in Fig. 2 the radial distribution function (RDF) for each bond type. In general, the location of the RDF peaks for all three bond types corresponds to the equilibrium Si-Si, Li-Si and Li-Li bond distances of 0.245, 0.265 , and $0.270 \mathrm{~nm}$, respectively, as shown by dashed lines. Interestingly, both the MD model structures created using the MEAM and ReaxFF potentials have quantitatively very similar RDF distributions, even though both these potentials were formulated on very different theoretical foundations. The spread of the RDF for Si-Si bonds is consistently very narrow $( \pm 0.02 \mathrm{~nm}$ ), indicating that Si-Si bonds are inherently stiff and have low stretchability of $\sim 8 \%$. The spread of the RDF for Li-Si bonds is much larger ( $\pm 0.04 \mathrm{~nm}$ ), with bond stretchability of $\sim 15 \%$. The spread of the RDF for $\mathrm{Li}-\mathrm{Li}$ bonds is the largest $( \pm 0.06 \mathrm{~nm})$, and is again consistent across all three $\mathrm{Li}_{x} \mathrm{Si}$ structures. This implies that $\mathrm{Li}-\mathrm{Li}$ bonds have the highest bond stretchability of $\sim 22 \%$. The location of the RDF peaks and distribution of the RDFs are both in close agreement with model structures created by our prior DFT study[18], with exceptions of the DFT-calculated RDFs for $\mathrm{Li}$-Li bonds of $\mathrm{LiSi}_{2}$ and $\mathrm{Si}$-Si bondsof $\mathrm{Li}_{15} \mathrm{Si}_{4}$ due to the limited number of $\mathrm{Li}$ and $\mathrm{Si}$ atoms in the respective DFT supercells.

We deform each of the above amorphous $\mathrm{Li}_{x} \mathrm{Si}$ model structures by stretching the simulation box in the out-of-plane $\left(x_{2}\right)$ direction, while rigidly constraining the in-plane dimensions of the box. MD simulations reveal that the deformation of $\mathrm{Li}_{x} \mathrm{Si}$ can be driven by a competition between cavitation induced by hydrostatic stress and shear yielding induced by deviatoric 
stress[30]. Here, our imposed uniaxial strain state subjects each $\mathrm{Li}_{x} \mathrm{Si}$ structure to combined hydrostatic and deviatoric stresses. This strain-controlled loading state was previously used in our prior DFT calculations[18], in favor of conventional (uniaxial) stress-controlled loading, to achieve more stable and complete stress-strain curves. This is especially important for $\mathrm{Li}_{x} \mathrm{Si}$ alloys with high Li content which undergo significant post-peak softening due to the nucleation and growth of nanopores. The use of the same loading state herefacilitates direct comparison between our MD and DFT results. In our DFT calculations, uniaxial straining was imposed quasi-statically by subjecting the supercell to incremental 2\% stretching in the out-of-plane direction, and allowing the atoms to relax to their equilibrium position quantum-mechanically after each increment. These calculations therefore resemble those from molecular statics at $0 \mathrm{~K}$. Similarly, we incrementallydeform our MD simulation box with $0.1 \%$ strain, by remapping all the atoms proportionally in the out-of-plane direction and allowing the atoms to relax to their local minimum energy configuration at $0 \mathrm{~K}$ before the next increment. This quasi-static loading

eliminates the effect of strain rate on the deformation response. We obtain the "macroscopic" stress-strain curve by averaging the atomic virial stresses of the $\mathrm{Li}$ and $\mathrm{Si}$ atoms over the entire deformed box volume. We also obtain the deformation response of the $\mathrm{Li}_{x} \mathrm{Si}$ alloysat $300 \mathrm{~K}$ by incrementallydeforming the structure with $0.1 \%$ strain, but equilibrating the structure at a target temperature of $300 \mathrm{~K}$ which is maintained by a Berendson thermostat for $0.5 \mathrm{ps}$ after each increment.

\section{Results}

To ascertain the accuracy of the MEAM and ReaxFF interatomic potentials in describing the deformation response of $\mathrm{Li}_{x} \mathrm{Si}$ alloys, wecompare the stress-strain curves obtained from MD simulations using these potentials against those from prior DFT calculations[18].Fig. 3 shows the von Mises stress-strain relationship for $\mathrm{LiSi}_{2}, \mathrm{LiSi}$, and $\mathrm{Li}_{15} \mathrm{Si}_{4}$ alloys obtained from MD simulations based on the MEAM potential. These stress-strain curvesare computed from MD model structures with edge dimensions of $d=4 \mathrm{~nm}$ at $0 \mathrm{~K}$, as well as $d=4,7$, and $15 \mathrm{~nm}$ at 300 K. Thecorresponding stress-strain results from DFT calculations are denoted by open symbols interconnected by dashed lines. The stress-strain curves from DFT display an initial linear elastic response followed by plastic yielding to reach peak stresses of 3, 2.5, and $1.5 \mathrm{GPa}$ for $\mathrm{LiSi}_{2}$, $\mathrm{LiSi}$, and $\mathrm{Li}_{15} \mathrm{Si}_{4}$, respectively. Beyond this point, the $\mathrm{LiSi}_{2}$ alloy undergoes sudden loss of stress- 
carryingcapacity which suggests catastrophic failure, while the $\mathrm{LiSi}$ and $\mathrm{Li}_{15} \mathrm{Si}_{4}$ alloys exhibit more gradual softening due to the cavitation and growth of nanopores. The stress-strain curves obtained from our MD simulations at $0 \mathrm{~K}$ for all three $\mathrm{Li}_{x} \mathrm{Si}$ alloys are remarkably in good agreement with DFT calculations. This suggests that the MEAM potential wellcaptures the theoretical elasto-plastic behavior of $\mathrm{Li}_{x} \mathrm{Si}$ alloys, although the MD simulations at $0 \mathrm{~K}$ are unable to capture the failure/softening response exhibited by DFT.At temperatures of $300 \mathrm{~K}$, the tensile yield stresses for all three $\mathrm{Li}_{x} \mathrm{Si}$ alloysare reduced by several-folds, and now exhibit a distinct loss of stress-carryingcapacity beyond a critical strain. Because of the statistical nature of the associated failure/cavitation process, this critical strain decreases with increasing model sized, at least up to a certain point,although d has no visible influence on the elasto-plastic properties. Therefore, a sufficiently large MD simulation box is needed to simulate the failure of $\mathrm{Li}_{x} \mathrm{Si}$ alloys. We further note that the critical strains for $\mathrm{d}=15 \mathrm{~nm}$ at $300 \mathrm{~K}$ are very close to those predicted by DFT calculations at 0 K.Fig. 4 compares the von Mises stress-strain relationship from MD simulations based on the ReaxFF potential versus DFT calculations for $\mathrm{LiSi}_{2}$, $\mathrm{LiSi}$, and $\mathrm{Li}_{15} \mathrm{Si}_{4}$ alloys. The ReaxFF-based MD simulations correctly predict the elastic softening and decreasing yield strength of $\mathrm{Li}_{x} \mathrm{Si}$ with increasing $\mathrm{Li}$ content. Unlike the MEAM-based MD results, however, the peak tensile stress values at $0 \mathrm{~K}$ obtained with ReaxFF are quantitatively different from those from DFT calculations. Specifically, the ReaxFF-based MD simulations at 0 $\mathrm{K}$ over-predict the peak yield stress values for $\mathrm{LiSi}_{2}$ and $\mathrm{LiSi}$ by $44 \%$ and $36 \%$, respectively, but accurately predict the yield stress value of $\sim 1.2 \mathrm{GPa}$ for $\mathrm{Li}_{15} \mathrm{Si}_{4}$.

We summarize in Fig. 5 the elastic modulus and peak tensile yield stressvalues versus the lithium fraction (defined as the ratio of $\mathrm{Li}$ atoms to the total number of atoms) of five $\mathrm{Li}_{x} \mathrm{Si}$ model structures obtained from our MEAM- and ReaxFF-based MD simulations conducted at 0 $\mathrm{K}$ and $300 \mathrm{~K}$. These results are based on our $\mathrm{d}=4 \mathrm{~nm} \mathrm{MD}$ simulation box, though we note that both the elastic modulus and yield stressare independent of d.To account for statistical variations, we conducted MD simulations to obtain the stress-strain response of 5 different amorphous $\mathrm{Li}_{x} \mathrm{Si}$ configurations. We find the error bars for both the elastic modulus and yield strength to be negligible for our $\mathrm{d}=4 \mathrm{~nm}$ model structure. We also include the results of DFT calculations[18], as well as those from experimental measurements[13,22,24,31], for comparisons with our $0 \mathrm{~K}$ and $300 \mathrm{~K}$ MD results respectively.Results from our ReaxFF- and MEAM-based MD simulations show that the elastic modulus and yield strength of $\mathrm{Li}_{x} \mathrm{Si}$ both decrease linearly with 
Li content and closely follow the rule-of-mixtures. In fact, the elastic modulus predictions from both potentials are very similar and are generally in good agreement with DFT calculations at 0 $\mathrm{K}$, but slightly under-predict the experimental values at $300 \mathrm{~K}$. The ReaxFF-based prediction of the tensile strength of pure Si at $0 \mathrm{~K}$ is in very good agreement with DFT calculations. We remark that pure amorphous Si does not actually undergo yielding but fails in a brittle manner without plastic deformation, and $\sigma_{y}$ here for pure Si represents the tensile strength. With this exception, the MEAM-based MD simulations are found to more accurately match the DFT results for lithiated $\mathrm{Si}$ at $0 \mathrm{~K}$. More importantly, the tensile yieldstrength predictions from these MEAM-based MD simulations at $300 \mathrm{~K}$ are also in perfect agreement with experimental yield strength measurements. In addition to the high accuracy of the MEAM potential, we find the computations based on this potential to be $\sim 40$ times faster than those based on ReaxFF potential.Our MD simulations in the following will be based on the MEAM potential.

In previous DFT calculations, it was concluded that the surprising plasticity behavior of $\mathrm{Li}_{x} \mathrm{Si}$, in contrast to the brittle behavior of pure $\mathrm{Si}$, is due to the nucleation and growth of nanopores[18].Because of the limited sizescale of the supercells in DFT, however, the observed porosity is in fact angstrom-sized voids caused by localized bond breaking. Using MD simulations, we seek to understand how these angstrom-scale voids develop into actual nanometer-scale voids, andhow these nanopores in turn grow and coalesce.Fig. 6 shows the evolution of porosity fversus theapplied strain $\epsilon_{22}$ for different MD model sizesof $\mathrm{d}=2.5,7,10$, 12, and $15 \mathrm{~nm}$. We again focus on $\mathrm{LiSi}_{2}, \mathrm{LiSi}$, and $\mathrm{Li}_{15} \mathrm{Si}_{4}$ alloys, which cover the spectrum of model structures with low, intermediate, and high $\mathrm{Li}$ content. For each alloy, we divide the deformed MD simulation box into regular grids, each of $(0.15)^{3} \mathrm{~nm}^{3}$. We consider each grid as a void if there are no atoms within a $0.3 \mathrm{~nm}$ radius, which is slightly larger than the equilibrium Li$\mathrm{Li}, \mathrm{Li}-\mathrm{Si}$ and $\mathrm{Si}-\mathrm{Si}$ bond distances. We then sum up the total voidvolume in the box, and divide this by the current deformed box volume to get $f$. For all three $\mathrm{Li}_{x} \mathrm{Si}$ alloys, the porosity versus strain curves become model-size-independent only with $d=12 \mathrm{~nm}$ and larger. For the $\mathrm{LiSi}_{2}$ alloy, the porosity remains negligible up to a critical strain of $\epsilon_{22}=0.2$, as seen for $\mathrm{d}=12$ or 15 $\mathrm{nm}$, upon which fdramatically increases indicating catastrophic brittle-like fracture; we remark that the use of smaller model sizes of $d=2.5 \mathrm{~nm}$ may lead to the incorrect interpretation of a more gradual failure response. For the LiSi alloy, the critical strain for the onset of pore growth 
occurs much earlier at $\epsilon_{22}=0.08$; the pore growth rate is also more gradual, suggesting a stable ductile failure response. For the fully-lithiated $\mathrm{Li}_{15} \mathrm{Si}_{4}$ alloy, nucleation of nanopores is delayed and initiates only at $\epsilon_{22}=0.12$. At this critical strain, the initial void growth rate is fairly rapid, though not catastrophic as in $\mathrm{LiSi}_{2}$; further loading slows down the pore growth rate to reach a steadystate similar to LiSi.

Next, we examine the nanopore nucleation, growth and coalescence process for $\mathrm{LiSi}_{2}, \mathrm{LiSi}$ and $\mathrm{Li}_{15} \mathrm{Si}_{4}$ alloys. We adopt relativelylarge $\mathrm{d}=15 \mathrm{~nm}$ MD model structureswhere the porosity evolution across all three alloys becomes model-sizeindependent (Fig. 6).For each of these model structures, we filter the deformed MD box to display only the voidswithin the structure, which are shaded grey in Figs. 7a-9a, a cross-sectional cut (dashed lines) of the corresponding atomic configurations and bonding structures in Figs. 7b-9b. For the $\mathrm{LiSi}_{2}$ alloy in Fig. 7,damage initiates at $\epsilon_{22}=0.195$. Within $1.5 \%$ strain increment, this damage spreads across the entire cross-section of the simulation box to cause cleavage fracture. The deformed atomic configurations at $\epsilon_{22}=0.195$ show the presence of incipient nanopores (angstrom-sized voids), which become more well-defined at $\epsilon_{22}=0.20$. Close-up view shows that these incipient nanopores originate from the breaking of Si-Li bonds, which result in the plasticity behavior of $\mathrm{LiSi}_{2}$ (Fig. 3a).Observe that theseincipient nanopores are well-separated by interconnected chains of Si-Si bonds, which prevent them from developing into true nanometer-sized pores. The Si-Si chains separating the incipient nanopores eventually break at $\epsilon_{22}=0.2$. Cracks subsequently form and propagate along the path which locally has the highest density of weaker Li-Si bonds, as evidenced by the broken Li and Si bonds along the sharp fracture surface.

For the LiSi alloy in Fig. 8, significant void growth already occurs at $\epsilon_{22}=0.12$, as shown by the presence of twoadjacent $\sim 2$ and $\sim 4 \mathrm{~nm}$ diameter nanopores within the structure. These nanopores stably grow, and coalesce at $\epsilon_{22}=0.14$. Theeventual formation of a void sheet at $\epsilon_{22}=0.16$ results in the softening stress-strain response (Fig. $3 \mathrm{~b}$ ). This ductile fracture process results in a much rougher fracture surface, as comparedto the brittle failure response of $\mathrm{LiSi}_{2}$. The corresponding cross-sectional views of the deformed atomic configurations (Fig. 8b), and the associated close-up views of the bonding configurations (Fig. 8c), show that the nanopores primarily growand coalesce by the breaking of $\mathrm{Li}-\mathrm{Li}$ and $\mathrm{Li}-\mathrm{Si}$ bonds, while the few $\mathrm{Si}$-Si bonds in the LiSi structure remain mostly intact. 
For the fully-lithiated $\mathrm{Li}_{15} \mathrm{Si}_{4}$ alloy in Fig. 9, a distinct $\sim 1 \mathrm{~nm}$ diameter nanopore first nucleates at $\epsilon_{22}=0.12$. Three additional nanopores of diameters $\sim 1-2 \mathrm{~nm}$ subsequently nucleate at $\epsilon_{22}=0.13$, each growing independently of one another. This delocalized growth of nanopores explains why the porosity evolution curves for $\mathrm{Li}_{15} \mathrm{Si}_{4}$ in Fig. 6c are not as strongly dependent on the model size $\mathrm{d}$ as compared to $\mathrm{LiSi}$ or $\mathrm{LiSi}_{2}$. The nanopores eventually coalesce at $\epsilon_{22}=0.14$, causing the loss of stress-carrying capacity for $\mathrm{Li}_{15} \mathrm{Si}_{4}$ (Fig. $\left.3 \mathrm{c}\right)$. The cross-sectional view of the deformed atomic configuration at $\epsilon_{22}=0.12$ shows distinct clusters of $\mathrm{Li}$ rich regions encapsulated by networks of $\mathrm{Si}-\mathrm{Si}$ and $\mathrm{Si}-\mathrm{Li}$ chains. This clustering of $\mathrm{Li}$ atoms is unlike our prior $\mathrm{Li}_{15} \mathrm{Si}_{4}$ structures obtained from DFT, wherethe $32 \mathrm{Si}$ atoms are relatively evenly-dispersed among the $120 \mathrm{Li}$ atoms due to the limited supercell size, as shown by the RDF peaks in Fig. 2. Close-up view shows that strain is localized within these Li-rich clusters, which are potential sites for nucleation of nanopores. Since these Li-rich clusters are evenly distributed throughout the entire MD simulation box, the damage process is more delocalized. At $\epsilon_{22}=0.13$, the breaking of Li-Li bonds within the Li-rich clusters results in the sudden formation of multiple discrete nanopores within the structure. While this causes a sudden spike in the porosity in Fig. 6c, the nanopores do not immediately coalesce because they are encapsulated by strong Si-Si and Si-Li bond networks. Instead, coalescence occurs gradually in a controlled fashion by the breaking of Li-Si bonds, as shown at $\epsilon_{22}=0.14$.

\section{Discussions}

Our above results demonstrate that the plasticity and cracking of $\mathrm{Li}_{x} \mathrm{Si}$ alloys are closelyrelated to the fraction of $\mathrm{Si}-\mathrm{Si}, \mathrm{Si}-\mathrm{Li}$, and $\mathrm{Li}-\mathrm{Li}$ bonds within the structure.At low $\mathrm{Li}$ concentrations, the peak tensile yield strength of the $\mathrm{Li}_{x} \mathrm{Si}$ alloy is at its highest, since the structure primarily comprises of covalent $\mathrm{Si}-\mathrm{Si}$ bonds. The breaking of weaker ionic $\mathrm{Li}$-Si bonds allows the alloy to undergo plasticity, but these broken Li-Si bonds are unable to fully-develop into nanopores because of the interconnected chains of $\mathrm{Si}-\mathrm{Si}$ bonds within the structure. The eventual scission of Si-Si bonds results in brittle fracture and the fracture surface remains very sharp due to the absence of significant void growth during plasticity. At intermediate Li concentrations, plasticity of the $\mathrm{Li}_{x} \mathrm{Si}$ alloy occurs by the breaking of $\mathrm{Li}-\mathrm{Si}$ bonds or/and the breaking of even weaker metallic $\mathrm{Li}-\mathrm{Li}$ bonds. Since the fraction of $\mathrm{Si}-\mathrm{Si}$ bonds is now smaller, the broken Li-Si or Li-Li bonds readily develop into nanopores, which explains the early onset of 
void growth in Fig. 6b. These nanopores in turn grow and coalesce to form a void sheet. This juncture at $x \sim 1$ marks the transition in the fracture behavior of $\mathrm{Li}_{x} \mathrm{Si}$ from brittle to ductile. At high $\mathrm{Li}$ concentrations, the $\mathrm{Li}_{x} \mathrm{Si}$ alloy is now able to undergo extreme deformations due to the high proportion of $\mathrm{Li}-\mathrm{Li}$ bonds, which have bond stretchability of $\sim 22 \%$ compared to $~ 15 \%$ and 8\% for Si-Li and Si-Si bonds (Fig. 2). This high bond stretchability delays the development of nanopores (compare Fig. 6c with Fig. 6b). Once these nanopores form resulting in the observed initial jump in porosity, subsequent void growth occurs more gradually (Fig. 6c). Each nanopore now grows independently of the surrounding nanopores, again because of the high Li-Li bond stretchability, which delays void coalescence and failure. Hence, the fully-lithiated $\mathrm{Li}_{15} \mathrm{Si}_{4}$ alloy will be considerably more ductile compared to LiSi.Much of the strength of this alloy now comes from the fewer Li-Si bonds within the structure, giving rise to the low tensile yield strength.

Our study demonstrates a sharp transition in the deformation behaviorof $\mathrm{Li}_{x} \mathrm{Si}$ from brittle to ductile as lithiation progresses. However, ductility and fracture toughness are two related, but different quantities, since the latter refers to the alloy's flaw tolerance. Are $\mathrm{Li}_{x} \mathrm{Si}$ alloys with high Li content truly more flaw-tolerant than those with low Li content? Our recent nanoindentation experiments appear to support this notion[17], though earlier experiments have alluded that the fracture toughness of $\mathrm{Li}_{x} \mathrm{Si}$ alloys is independent of $x[16]$. Here, we introduce an initial flaw in the form of a $1.5 \mathrm{nmby} 0.5 \mathrm{~nm}$ through-crack located in the center of the periodic MD simulation box of $\mathrm{d}=15 \mathrm{~nm}$, for the $\mathrm{LiSi}_{2}, \mathrm{LiSi}$, and $\mathrm{Li}_{15} \mathrm{Si}_{4}$ model structures. To prevent the flaw from closing, we impose an initial uniaxial strain of $5 \%$ by proportionally displacing the atoms in the simulation box and allowing the system to relax at the temperature of $300 \mathrm{~K}$. Thereafter, we continue deforming each $\mathrm{Li}_{x} \mathrm{Si}$ structure, and show snapshots of the atomic configurations at various instants in Fig. 10. At $\epsilon_{22}=0.1$, the initial sharp flaw becomes oblate for $\mathrm{LiSi}_{2}$, but is distinctly more circular for $\mathrm{Li}_{15} \mathrm{Si}_{4}$. At $\epsilon_{22}=0.2$, crack propagates through and separates the entire $\mathrm{LiSi}_{2}$ structure, but only propagates partially through the LiSi structure. For the fullylithiated $\mathrm{Li}_{15} \mathrm{Si}_{4}$ structure, however, the flaw now becomes a cylindrical voidand continues to grow with no signs of necking. We attribute this high flaw tolerance of $\mathrm{Li}_{15} \mathrm{Si}_{4}$ alloys to the large proportion of Li-Li bonds within the structure which can tolerate high stretches of $~ 22 \%$ without breaking.Therefore, these $\mathrm{MD}$ simulations demonstrate that the flaw tolerance of $\mathrm{Li}_{x} \mathrm{Si}$ alloys indeed increases with $x$, which is in agreement with our recent experimental results[17]. 
We remark that the MD simulations conducted here have provided mechanistic insights into the plasticity and cracking behavior of $\mathrm{Li}_{x} \mathrm{Si}$ alloys at scales not within the capabilities of DFT. This opens up unique possibilities for future simulation-based nanostructured electrode design. The accuracy of these MD simulations hinges on the interatomic potentials. The bulk of our current MD studies was carried out using the MEAM potential by Cui et al.[27]. This MEAM potential was found to be highly accurate in replicating the elasto-plastic responses from DFT calculations at $0 \mathrm{~K}$ and experimental measurements at $300 \mathrm{~K}$ (Fig. 5). More impressively, the associated bond-breaking mechanisms from these MD simulations were found to be in very good agreement with our prior DFT studies[18]. In comparison, the MD simulations based on the ReaxFF potential by Ostadhossein et al.[26] tend to over-predict the yield strengths of $\mathrm{Li}_{x} \mathrm{Si}$ alloys (Fig. 5b). Nevertheless, the ReaxFF potential is specifically designed to account for chemical bond breaking and bond formation processes, and we find the underlying plasticity and cracking mechanisms associated with the ReaxFF potential to be largely similar to those from our MEAM-based MD simulations.

\section{Conclusion}

In summary, MD simulations have provided quantitative insights into the deformation behavior of $\mathrm{Li}_{x} \mathrm{Si}$ alloys. The MEAM-based MD simulations at $0 \mathrm{~K}$ correctly reproduce the stress-strain response of $\mathrm{Li}_{x} \mathrm{Si}$ alloys from DFT calculations, while matching the yield strength data from experiments at $300 \mathrm{~K}$. These MD simulations demonstrate the brittle-to-ductile transition of $\mathrm{Li}_{x} \mathrm{Si}$ alloys, as well as the increasing flaw tolerance, with increasing Li content. This transition is largely associated with increasing proportion of highly-stretchable Li-Li bonds at the expense of stiffer Si-Si bonds. In addition, these simulations have identified the breaking of $\mathrm{Li}-\mathrm{Li}$ and/or Li-Si bonds as the precursor for nanopore nucleation and growth. These atomistic mechanisms fullyexplain the macroscopic plasticity and fracture behavior of $\mathrm{Li}_{x} \mathrm{Si}$ alloys.

\section{Acknowledgement}

The authors acknowledge the support of National Science Foundation Grant No. NSF-CMMI1300805, as well as computational time provided by TACC Grant No. TG-MSS130007, and Blue Waters sustained-petascale computing project which is supported by the National Science Foundation (Award Nos. OCI-0725070 and ACI-1238993) and the state of Illinois. Blue Waters 
is a joint effort of the University of Illinois at Urbana-Champaign and its National Center for Supercomputing Applications.

\section{References}

[1] H. Gasteiger. Lithium batteries advanced technologies and applications. 1st ed., John Wiley \& Sons, Inc., Hoboken, New Jersey, 2013.

[2] L.Y. Beaulieu, K.W. Eberman, R.L. Turner, L.J. Krause, J.R. Dahn. Colossal reversible volume changes in lithium alloys, Electrochem. Solid State Lett 4 (2001) A137-A140.

[3] J.P. Maranchi, A.F. Hepp, A.G. Evans, N.T. Nuhfer, P.N. Kumta. Interfacial properties of the a$\mathrm{Si} / \mathrm{Cu}$ : active-inactive thin-film anode system for lithium-ion batteries, J. Electrochem. Soc. 153 (2006) A1246-A1253.

[4] H.B. Chew, B. Hou, X. Wang, S. Xia. Cracking mechanisms in lithiated silicon thin film electrodes, Int. J. Solids Struct. 51 (2014) 4176-4187.

[5] H. Wang, B. Hou, X. Wang, S. Xia, H.B. Chew. Atomic-Scale Mechanisms of Sliding along an Interdiffused Li-Si-Cu Interface, Nano Lett. 15 (2015) 1716-1721.

[6] S.W. Lee, M.T. McDowell, L.A. Berla, W.D. Nix, Y. Cui. Fracture of crystalline silicon nanopillars during electrochemical lithium insertion, Proc. Natl. Acad. Sci. U.S.A. 109 (2012) 4080-4085.

[7] X. Liu, L. Zhong, S. Huang, S.X. Mao, T. Zhu, J. Huang. Size-Dependent Fracture of Silicon Nanoparticles During Lithiation, ACS Nano 6 (2012) 1522-1531.

[8] J. Graetz, C.C. Ahn, R. Yazami, B. Fultz. Highly reversible lithium storage in nanostructured silicon, Electrochem. Solid State Lett. 6 (2003) A194-A197.

[9] T. Takamura, S. Ohara, M. Uehara, J. Suzuki, K. Sekine. A vacuum deposited Si film having a Li extraction capacity over 2000 mAh/g with a long cycle life, J. Power Sources 129 (2004) 96-100.

[10] N. Liu, H. Wu, M.T. McDowell, Y. Yao, C.M. Wang, Y. Cui. A Yolk-Shell Design for Stabilized and Scalable Li-Ion Battery Alloy Anodes, Nano Lett. 12 (2012) 3315-3321.

[11] Q. Xiao, M. Gu, H. Yang, B. Li, C. Zhang, Y. Liu, F. Liu, F. Dai, L. Yang, Z. Liu, X. Xiao, G. Liu, P. Zhao, S. Zhang, C. Wang, Y. Lu, M. Cai. Inward lithium-ion breathing of hierarchically porous silicon anodes, Nat. Commun. 6 (2015) 8844.

[12] K. Zhao, W. Wang, J. Gregoire, M. Pharr, Z. Suo, J.J. Vlassak, E. Kaxiras. Lithium-Assisted Plastic Deformation of Silicon Electrodes in Lithium-Ion Batteries: A First-Principles Theoretical Study, Nano Lett. 11 (2011) 2962-2967.

[13] V.A. Sethuraman, M.J. Chon, M. Shimshak, V. Srinivasan, P.R. Guduru. In situ measurements of stress evolution in silicon thin films during electrochemical lithiation and delithiation, J. Power Sources 195 (2010) 5062-5066.

[14] V.A. Sethuraman, V. Srinivasan, A.F. Bower, P.R. Guduru. In Situ Measurements of StressPotential Coupling in Lithiated Silicon, J. Electrochem. Soc. 157 (2010) A1253-A1261.

[15] M.J. Chon, V.A. Sethuraman, A. McCormick, V. Srinivasan, P.R. Guduru. Real-Time Measurement of Stress and Damage Evolution during Initial Lithiation of Crystalline Silicon, Phys. Rev. Lett. 107 (2011) 045503.

[16] M. Pharr, Z. Suo, J.J. Vlassak. Measurements of the Fracture Energy of Lithiated Silicon Electrodes of Li-Ion Batteries, Nano Lett. 13 (2013) 5570-5577.

[17] X. Wang, F. Fan, J. Wang, H. Wang, S. Tao, A. Yang, Y. Liu, H.B. Chew, S.X. Mao, T. Zhu, S. Xia. High damage tolerance of electrochemically lithiated silicon, Nat. Commun. 6 (2015) 8417.

[18] H. Wang, X. Wang, S. Xia, H.B. Chew. Brittle-to-ductile transition of lithiated silicon electrodes: Crazing to stable nanopore growth, J. Chem. Phys. 143 (2015) 104703. 
[19] A.F. Bower, P.R. Guduru, V.A. Sethuraman. A finite strain model of stress, diffusion, plastic flow, and electrochemical reactions in a lithium-ion half-cell, J. Mech. Phys. Solids 59 (2011) 804-828.

[20] L. Brassart, Z. Suo. Reactive flow in solids, J. Mech. Phys. Solids 61 (2013) 61-77.

[21] X. Liu, H. Zheng, L. Zhong, S. Huan, K. Karki, L. Zhang, Y. Liu, A. Kushima, W. Liang, J. Wang, J.H. Cho, E. Epstein, S.A. Dayeh, S.T. Picraux, T. Zhu, J. Li, J.P. Sullivan, J. Cumings, C. Wang, S.X. Mao, Z. Ye, S. Zhang, J. Huang. Anisotropic Swelling and Fracture of Silicon Nanowires during Lithiation, Nano Lett. 11 (2011) 3312-3318.

[22] B. Hertzberg, J. Benson, G. Yushin. Ex-situ depth-sensing indentation measurements of electrochemically produced Si-Li alloy films, Electrochem. Commun. 13 (2011) 818-821.

[23] V.B. Shenoy, P. Johari, Y. Qi. Elastic softening of amorphous and crystalline Li-Si Phases with increasing Li concentration: A first-principles study, J. Power Sources 195 (2010) 6825-6830.

[24] A. Kushima, J.Y. Huang, J. Li. Quantitative Fracture Strength and Plasticity Measurements of Lithiated Silicon Nanowires by In Situ TEM Tensile Experiments, ACS Nano 6 (2012) 9425-9432.

[25] K. Zhao, G.A. Tritsaris, M. Pharr, W.L. Wang, O. Okeke, Z. Suo, J.J. Vlassak, E. Kaxiras. Reactive Flow in Silicon Electrodes Assisted by the Insertion of Lithium, Nano Lett. 12 (2012) 4397-4403.

[26] A. Ostadhossein, E.D. Cubuk, G.A. Tritsaris, E. Kaxiras, S.L. Zhang, A.C.T. van Duin. Stress effects on the initial lithiation of crystalline silicon nanowires: reactive molecular dynamics simulations using ReaxFF, Phys. Chem. Chem. Phys. 17 (2015) 3832-3840.

[27] Z. Cui, F. Gao, Z. Cui, J. Qu. A second nearest-neighbor embedded atom method interatomic potential for Li-Si alloys, J. Power Sources 207 (2012) 150-159.

[28] S. Plimpton. Fastparallelalgorithmsfor short-rangemolecular-dynamics, J. Comput. Phys. 117 (1995) 1-19.

[29] M.I. Baskes. Modifiedembedded-atompotentialsfor cubic materialsand impurities, Phys. Rev. B 46 (1992) 2727-2742.

[30] B. Ding, X. Li, X. Zhang, H. Wu, Z. Xu, H. Gao. Brittle versus ductile fracture mechanism transition in amorphous lithiated silicon: From intrinsic nanoscale cavatation to shear banding, Nano Energy 18 (2015) 8.

[31] L.A. Berla, S.W. Lee, Y. Cui, W.D. Nix. Mechanical behavior of electrochemically lithiated silicon, J. Power Sources 273 (2015) 41-51. 


\section{Figure Captions}

FIG. 1. Final relaxed atomic configurations of amorphous $\mathrm{Si}, \mathrm{LiSi}{ }_{2}, \mathrm{LiSi}, \mathrm{Li}_{12} \mathrm{Si}_{7}$ and $\mathrm{Li}_{15} \mathrm{Si}_{4}$ after rapid heating and quenching in MD. The simulations are conducted using $\sim 4 \times 4 \times 4 \mathrm{~nm}^{3} \mathrm{MD}$ simulation boxes, and are based on the (a) MEAM and (b) ReaxFF interatomic potentials. The Si and Li atoms are colored in orange and yellow, respectively.

FIG. 2. Comparison of the RDF distributions for Si-Si, $\mathrm{Li}-\mathrm{Si}$, and $\mathrm{Li}-\mathrm{Li}$ bonds within $\mathrm{LiSi}_{2}, \mathrm{LiSi}$, and $\mathrm{Li}_{15} \mathrm{Si}_{4}$ alloys, created by MD simulations based on the ReaxFF (red) and MEAM potentials (blue), as well as prior DFT calculations (black). Dashed vertical lines denote the equilibrium bond distance for the corresponding bond type.

FIG. 3. Comparison of the vonMises effective stress-strain response for (a) $\mathrm{LiSi}_{2}$, (b) $\mathrm{LiSi}$, and (c) $\mathrm{Li}_{15} \mathrm{Si}_{4}$ from MD simulations based on the MEAM potential versus DFT calculations.

FIG. 4. Comparison of the vonMises effective stress-strain response for (a) $\mathrm{LiSi}_{2}$, (b) $\mathrm{LiSi}$, and (c) $\mathrm{Li}_{15} \mathrm{Si}_{4}$ from MD simulations based on the ReaxFF potential versus DFT calculations.

FIG. 5. Summary of elastic modulus and tensile yield strength of $\mathrm{Li}_{x} \mathrm{Si}$ alloys at different lithium fractions from MEAM- and ReaxFF-based MD simulations, DFT calculations, and experiments.

FIG. 6. Simulation box size effects d on the porosity evolution versus applied strain for (a) $\mathrm{LiSi}_{2}$, (b) LiSi, and (c) $\mathrm{Li}_{15} \mathrm{Si}_{4}$ alloys.

FIG. 7. Cleavage fracture process for $\mathrm{LiSi}_{2}$. (a) Porosity evolution within the model structure at three instances. (b) Cross-sectional views of the atomic configurations taken along the dashed lines in (a). The Si and Li atoms are colored in orange and yellow, respectively.

FIG. 8. Ductile fracture process for LiSi. (a) Porosity evolution within the model structure at three instances. (b) Cross-sectional views of the atomic configurations taken along the dashed lines in (a). (c) Close-up view of the Li-Li and Li-Si bond breaking process. The Si and Li atoms are colored in orange and yellow, respectively. 
FIG. 9. Ductile fracture process for $\mathrm{Li}_{15} \mathrm{Si}_{4}$. (a) Porosity evolution within the model structure at three instances. (b) Cross-sectional views of the atomic configurations taken along the dashed lines in (a). The Si and Li atoms are colored in orange and yellow, respectively.

FIG. 10. Snapshots of the atomic configurations for (a) $\mathrm{LiSi}_{2}$, (b) $\mathrm{LiSi}$, and (c) $\mathrm{Li}_{15} \mathrm{Si}_{4}$, each with the same pre-existing flaw within the model structure, demonstrating increasing flaw tolerance with $\mathrm{Li}$ content. The Si and $\mathrm{Li}$ atoms are colored in orange and yellow, respectively. 
$\mathrm{Si}$

$\mathrm{LiSi}_{2}$

a
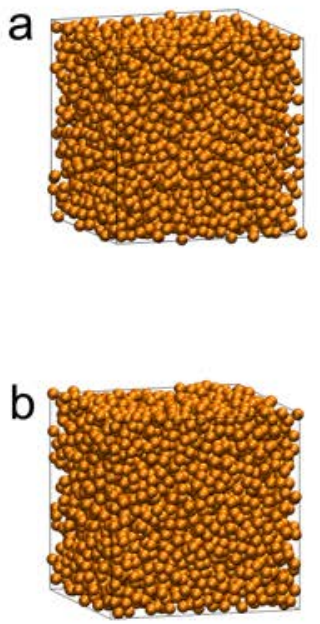

LiSi
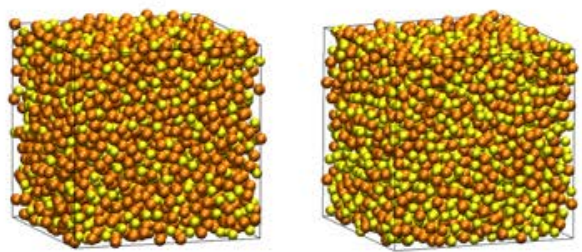

$\mathrm{Li}_{12} \mathrm{Si}_{7}$

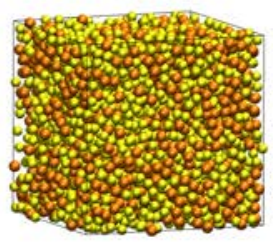

$\mathrm{Li}_{15} \mathrm{Si}_{4}$

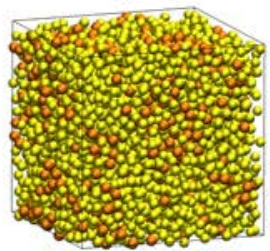

MEAM
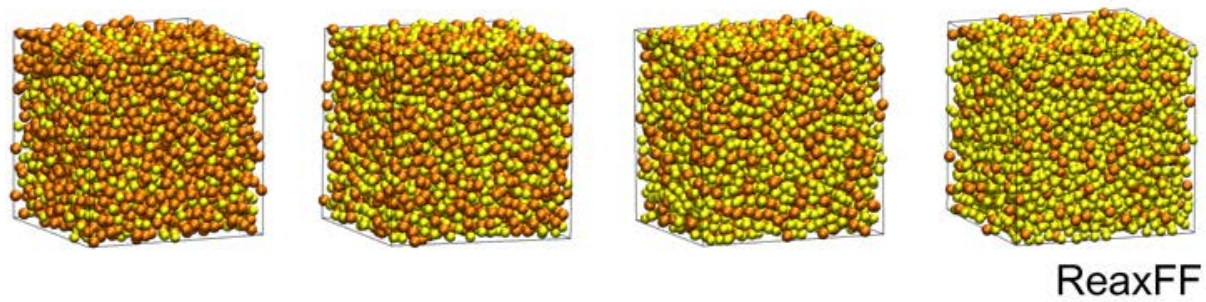

- Si

- $\mathrm{Li}$

Figure 1 

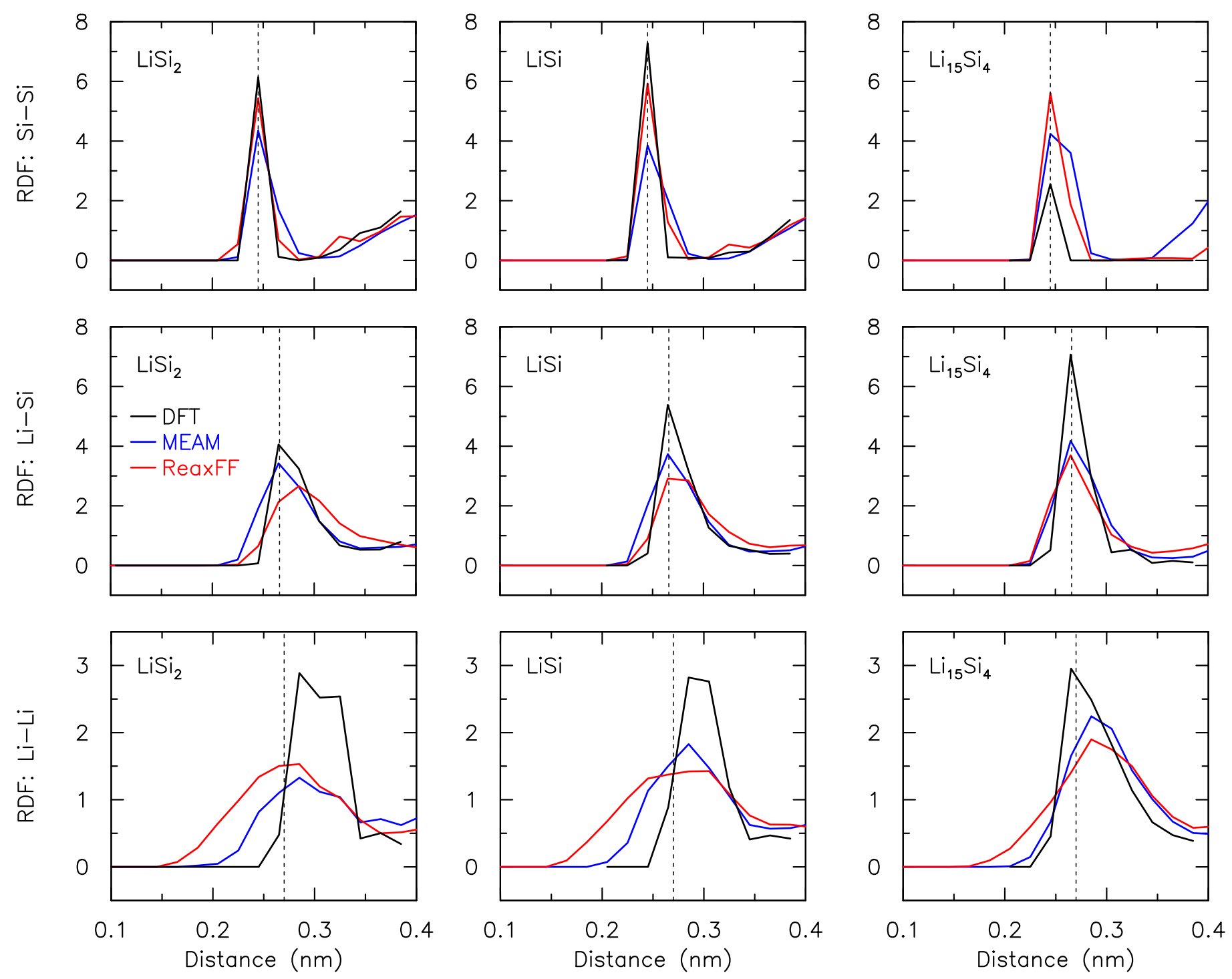

Figure 2 


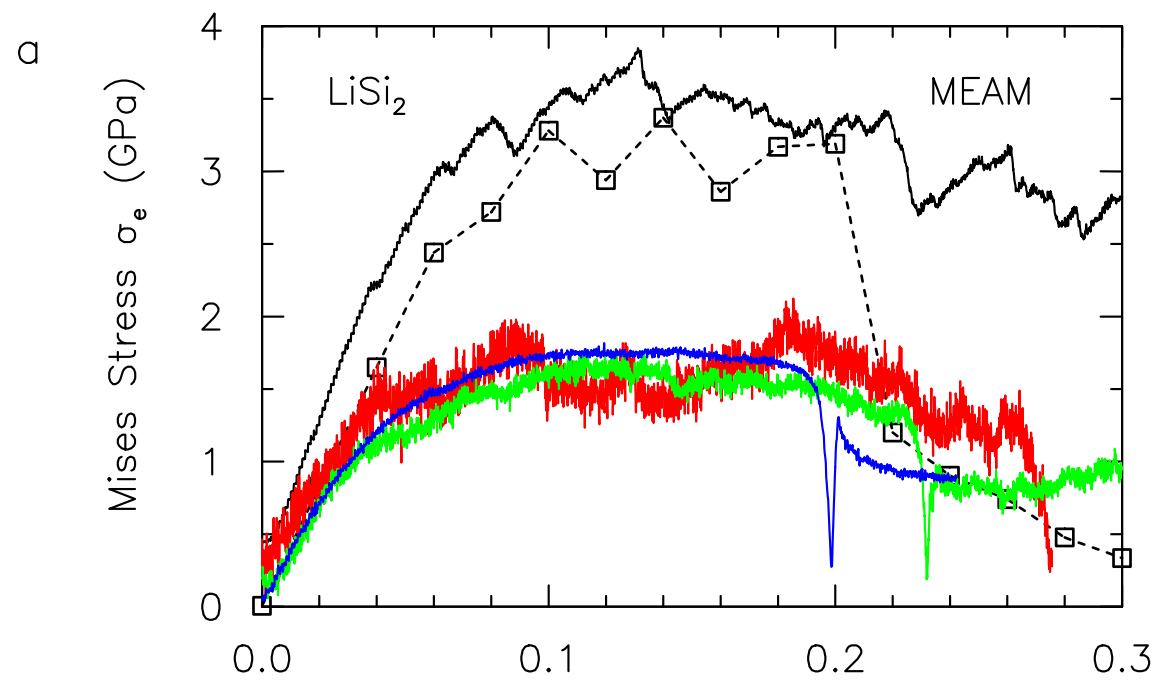

b

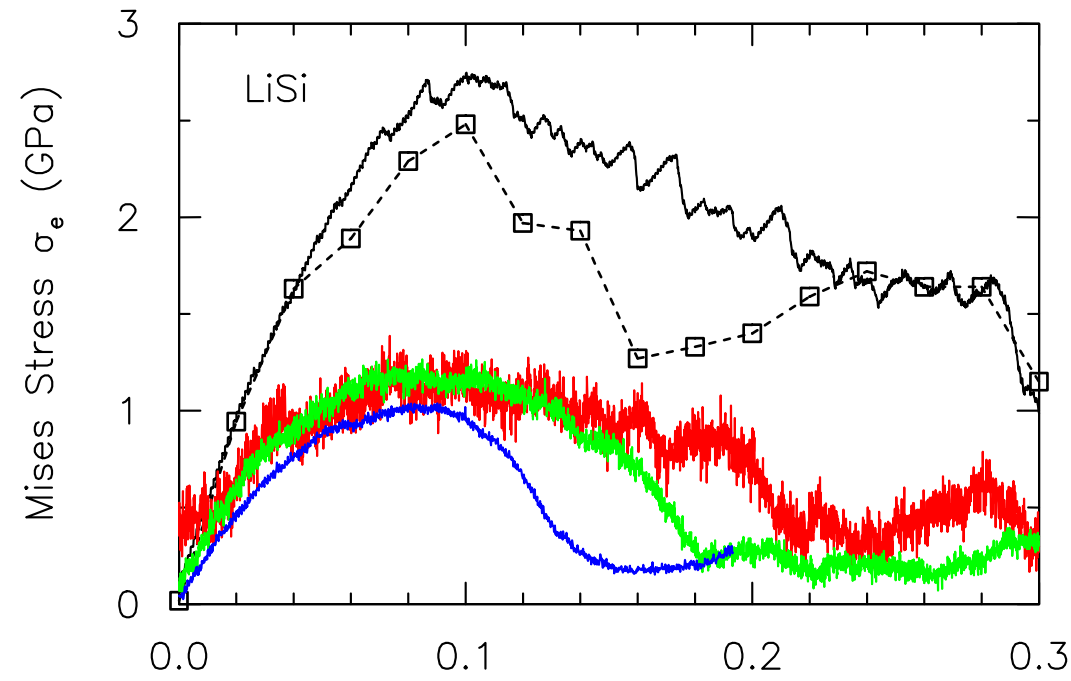

C

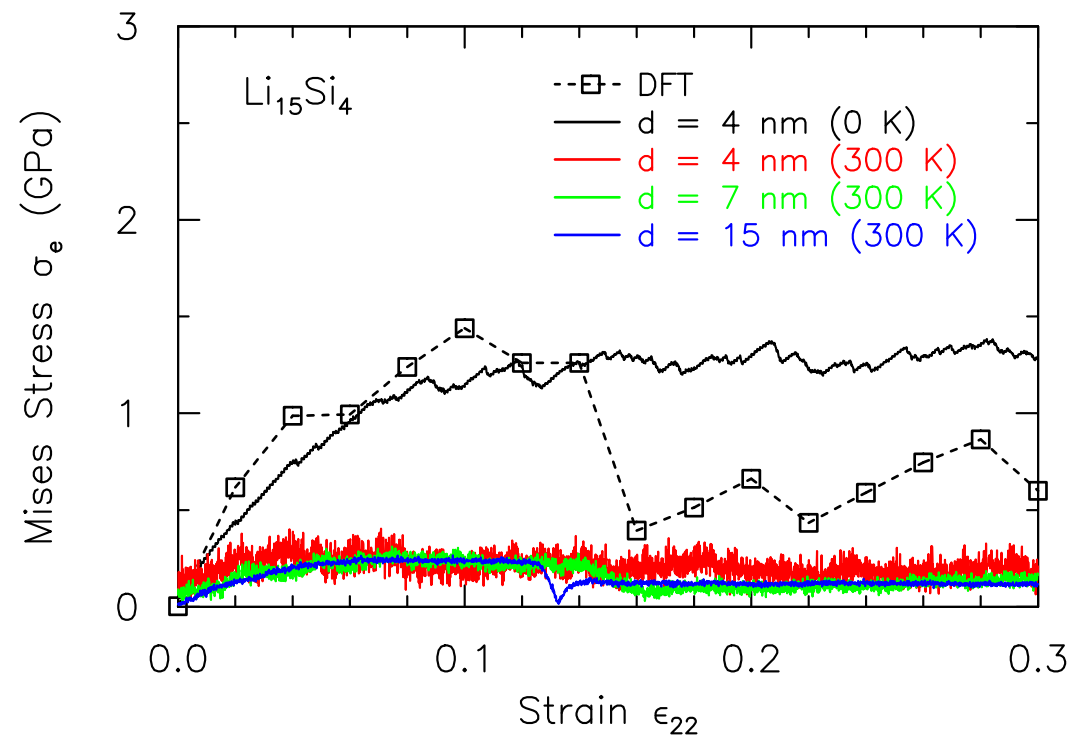

Figure 3 


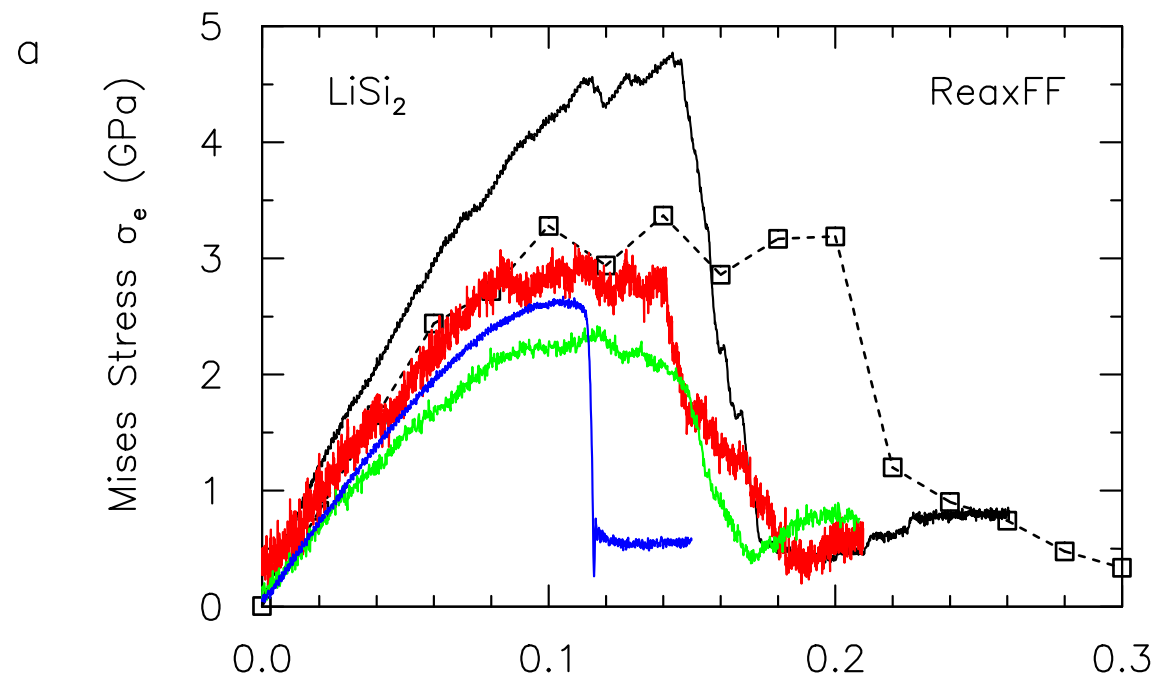

b

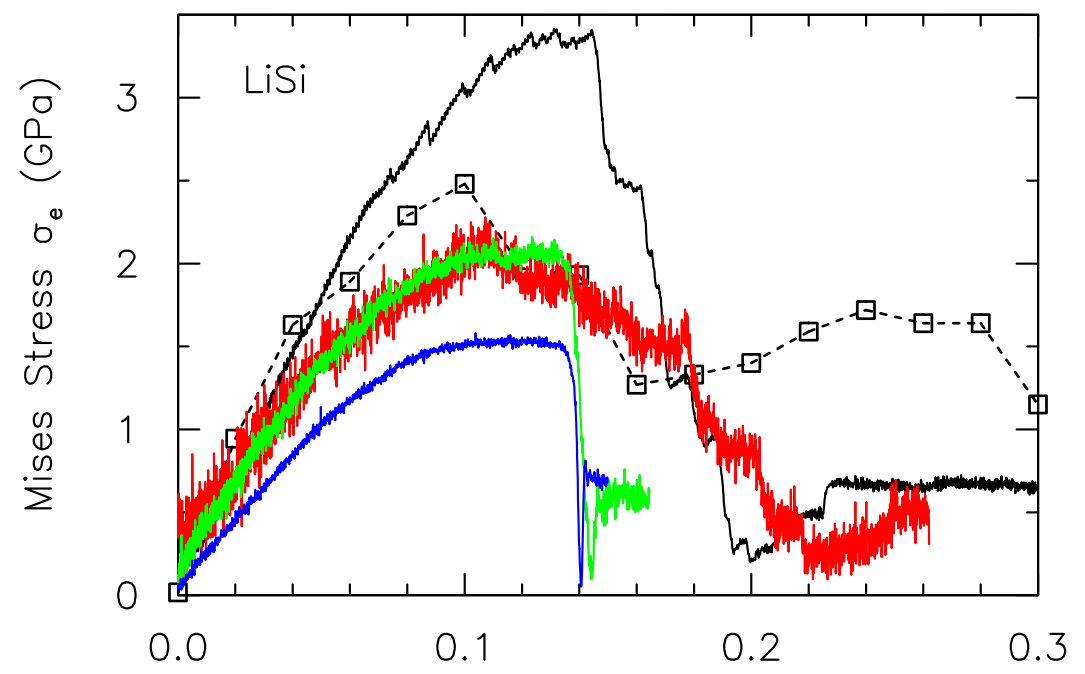

C

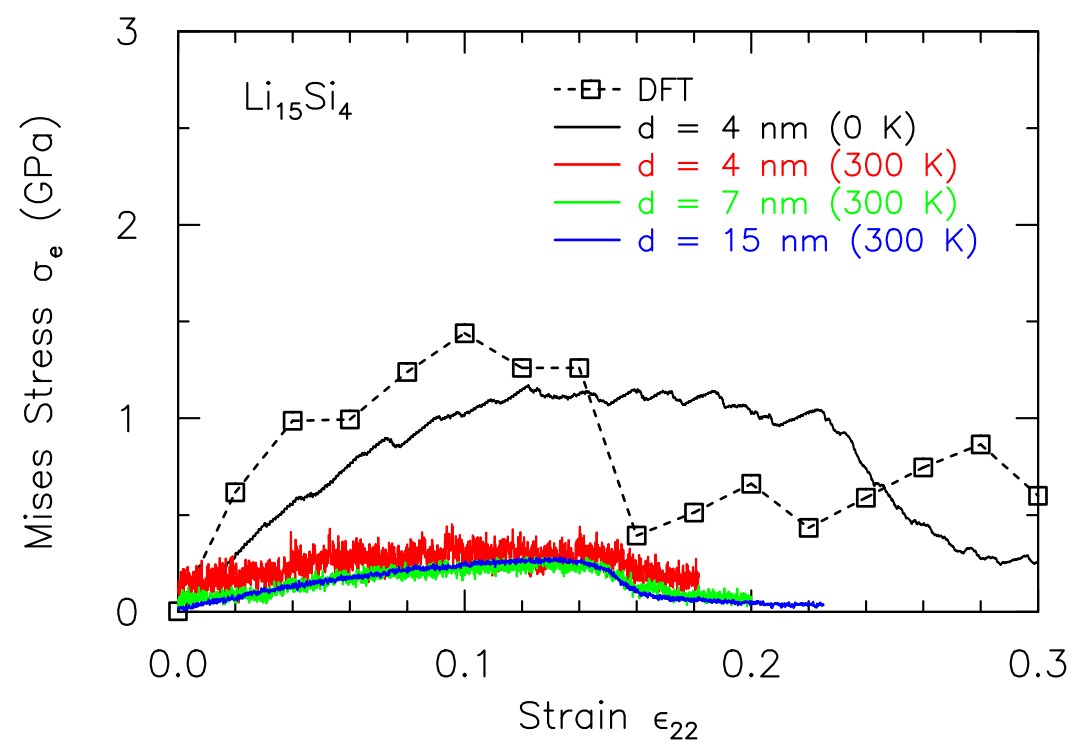

Figure 4 


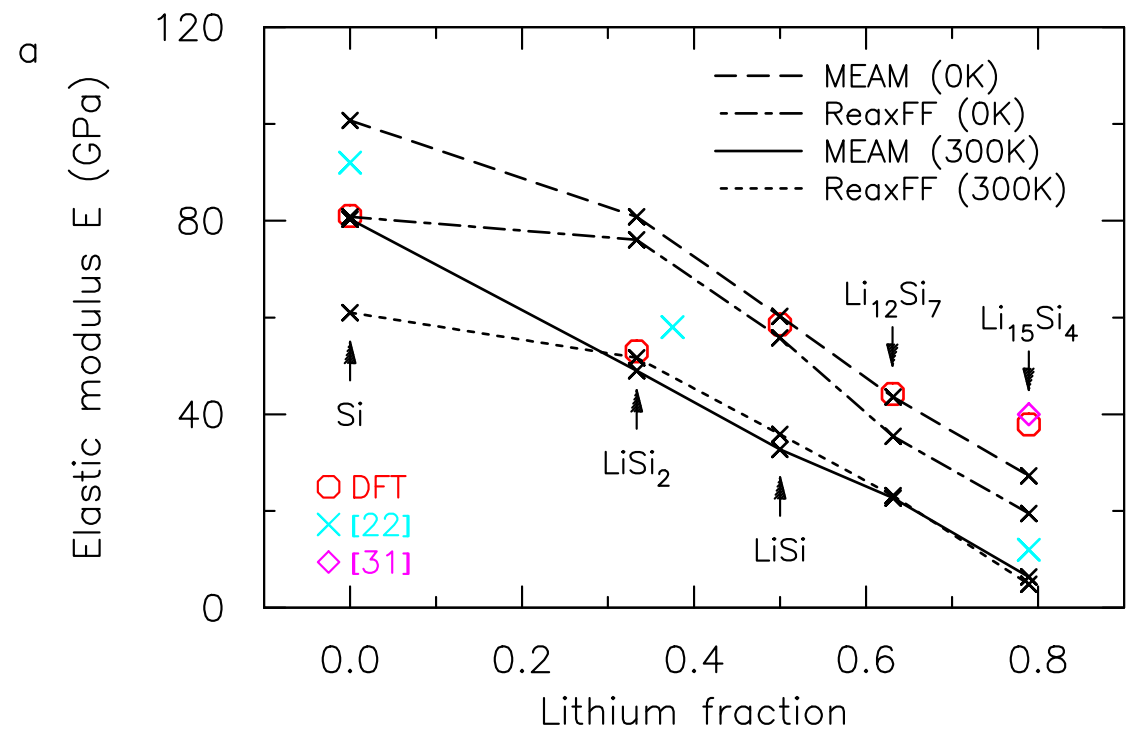

b

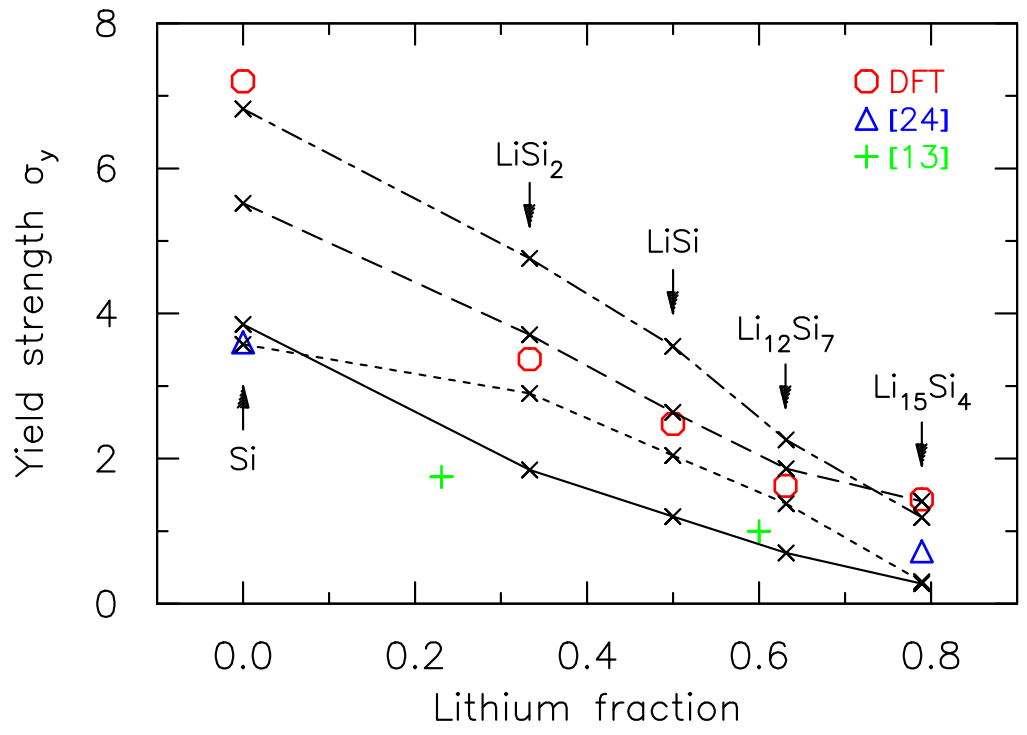

Figure 5 
a

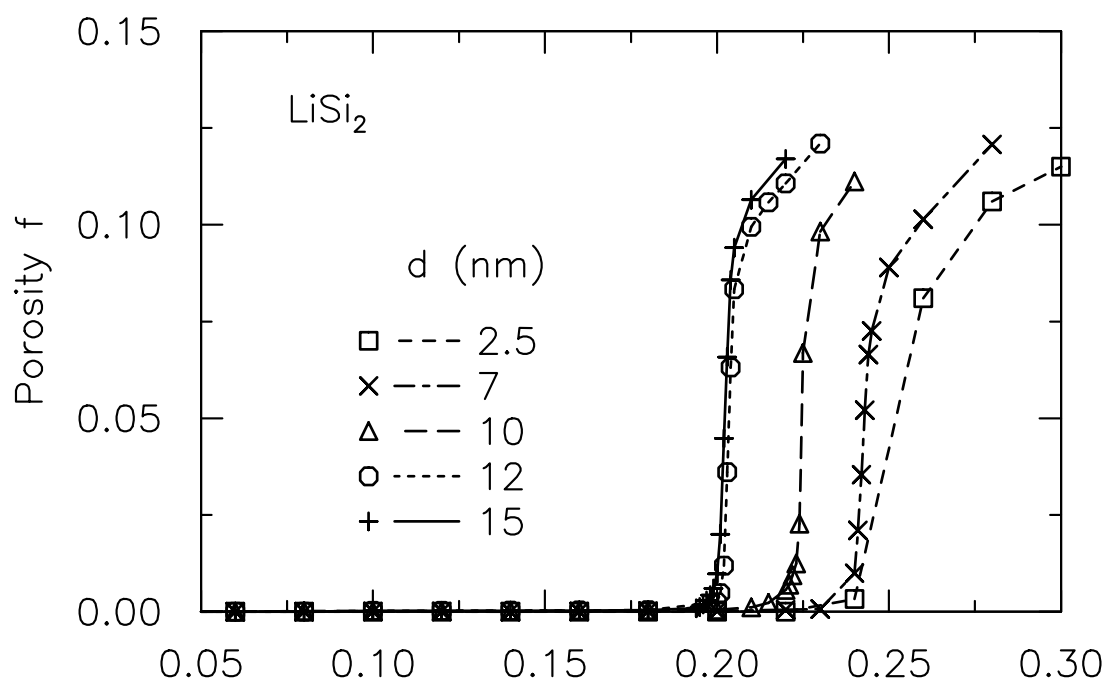

b

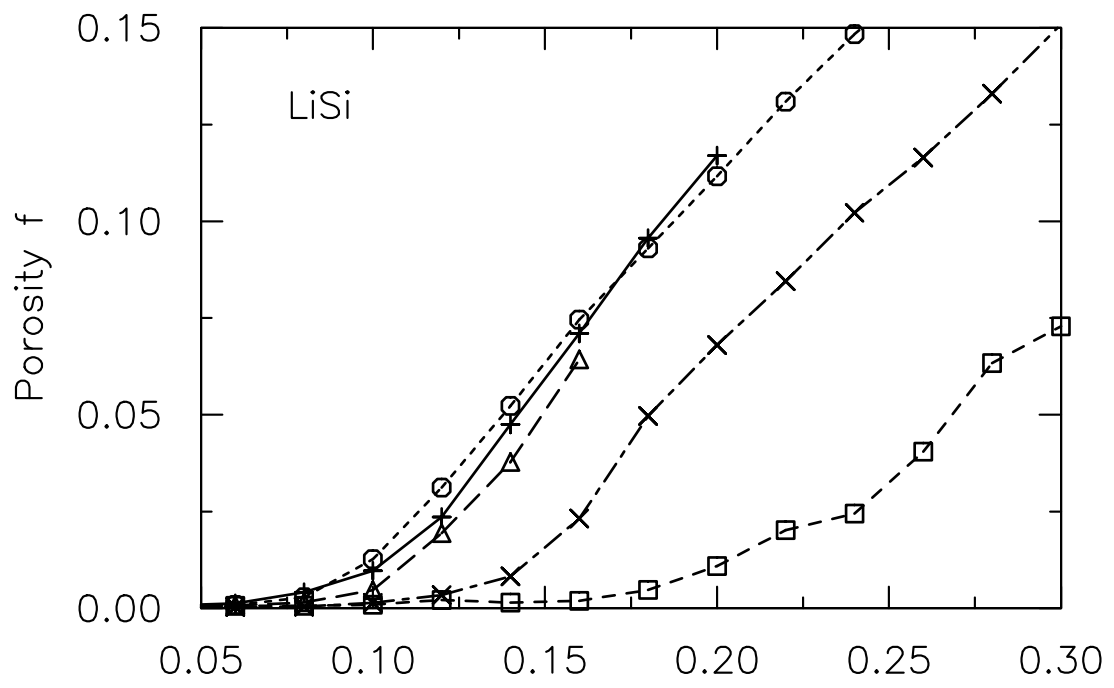

c

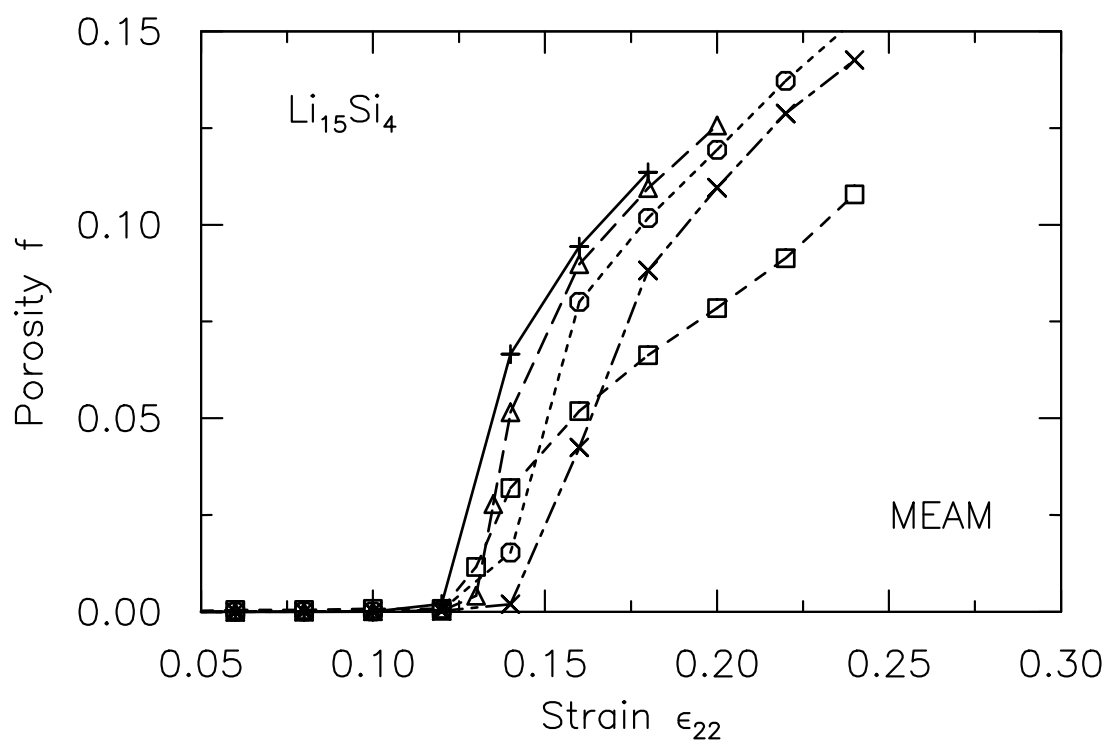

Figure 6 
a

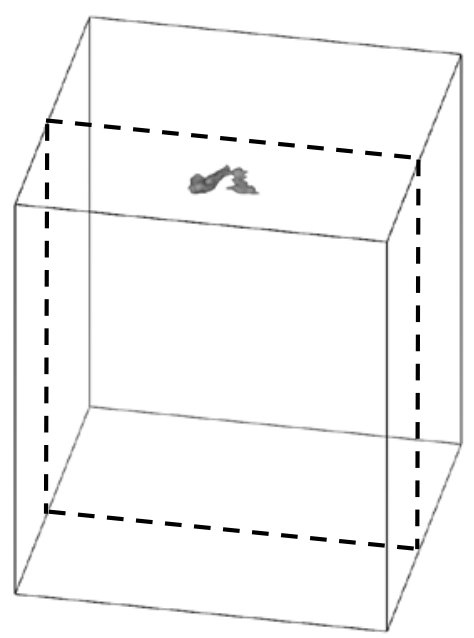

$\epsilon_{22}=0.195$

b

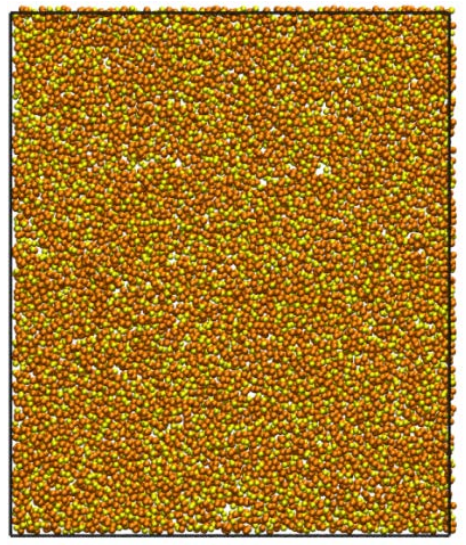

$\epsilon_{22}=0.195$

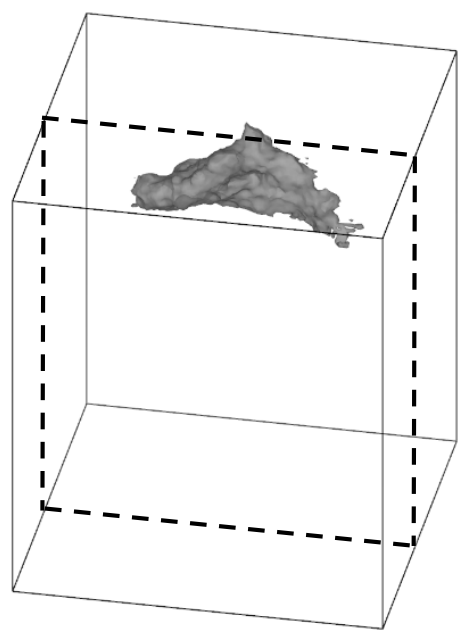

$\epsilon_{22}=0.20$

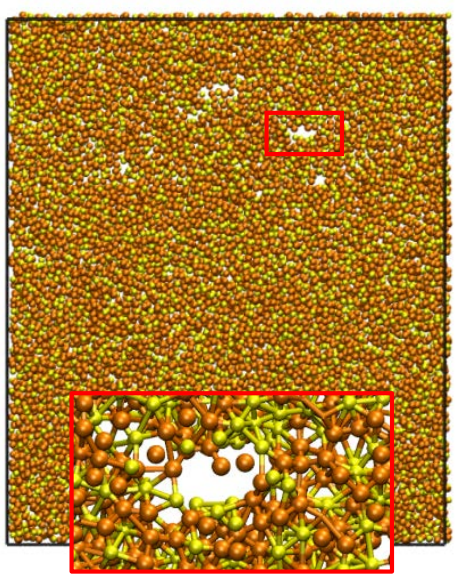

$\epsilon_{22}=0.20$

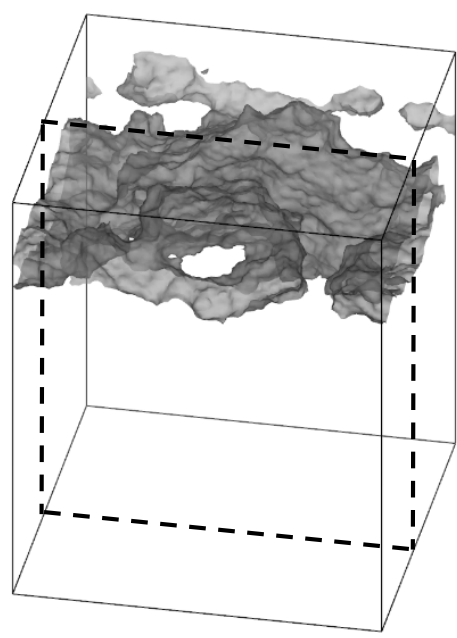

$\epsilon_{22}=0.21$

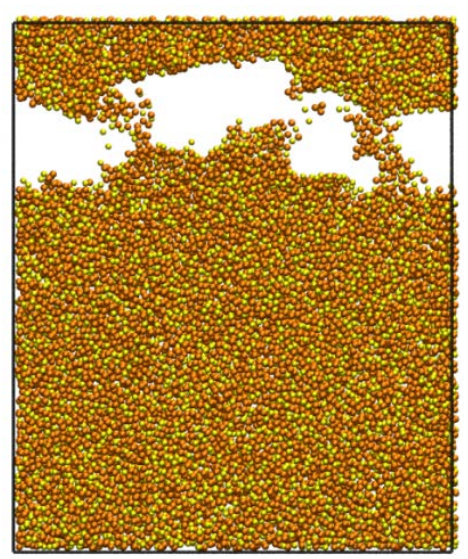

$\epsilon_{22}=0.21$

Figure 7 
a

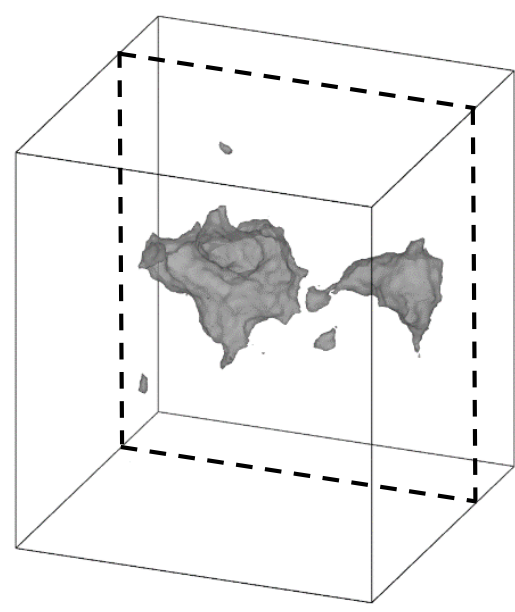

$$
\epsilon_{22}=0.12
$$

b

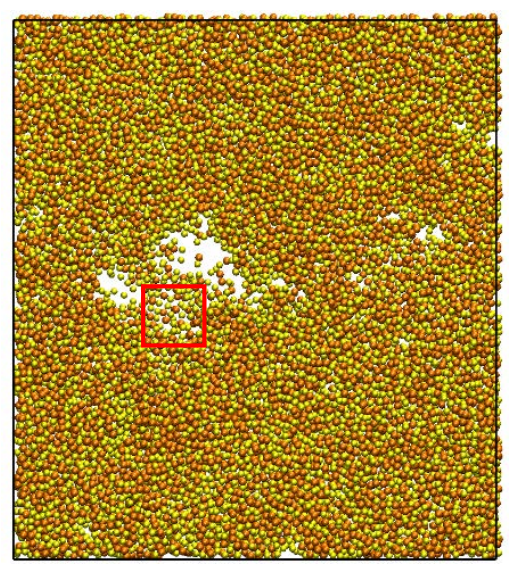

$$
\epsilon_{22}=0.12
$$

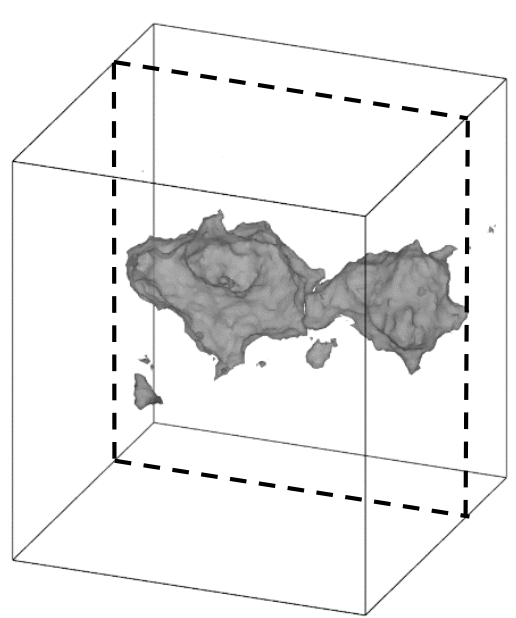

$\epsilon_{22}=0.14$

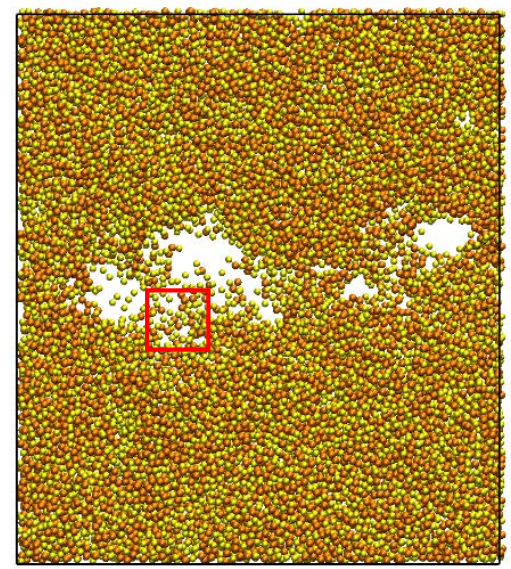

$\epsilon_{22}=0.14$

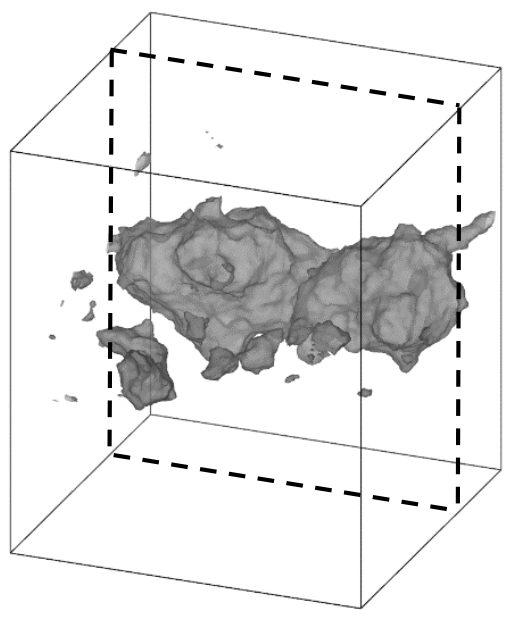

$\epsilon_{22}=0.16$

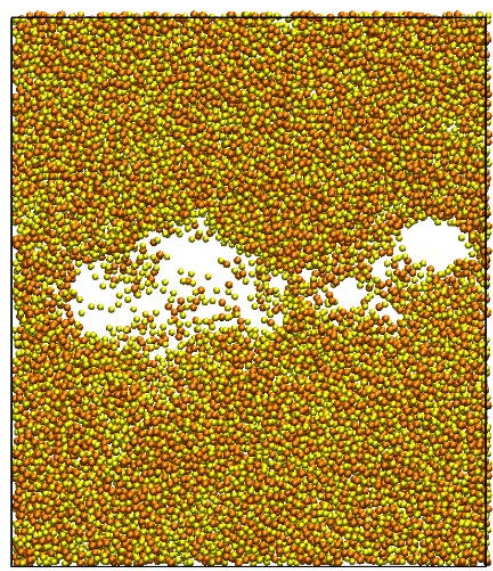

$\epsilon_{22}=0.16$
C

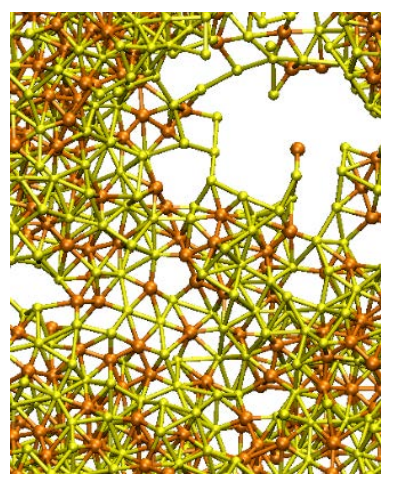

$$
\epsilon_{22}=0.11
$$

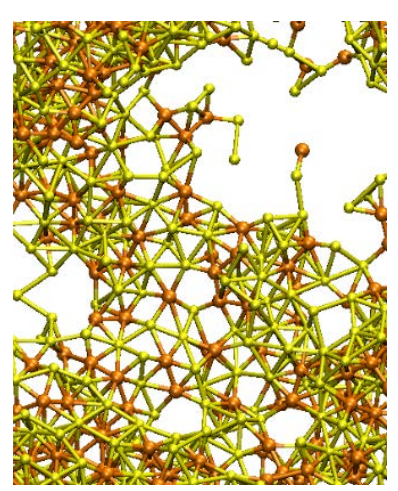

$\epsilon_{22}=0.12$

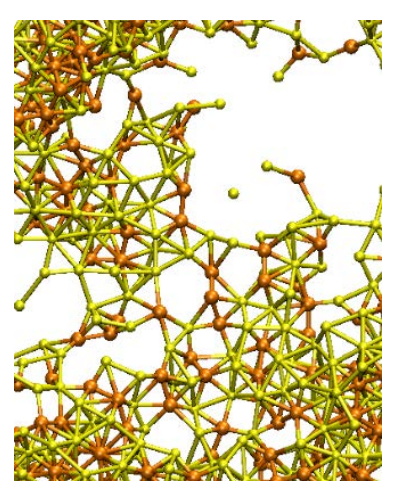

$\epsilon_{22}=0.13$

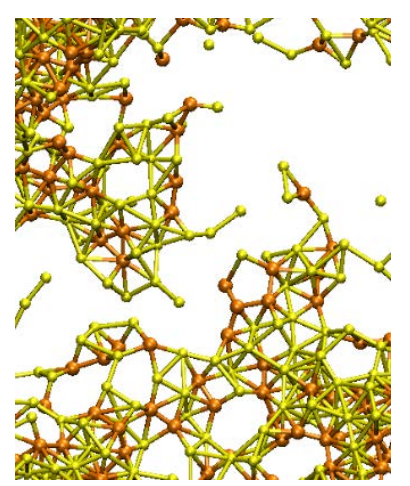

$\epsilon_{22}=0.14$ 
a

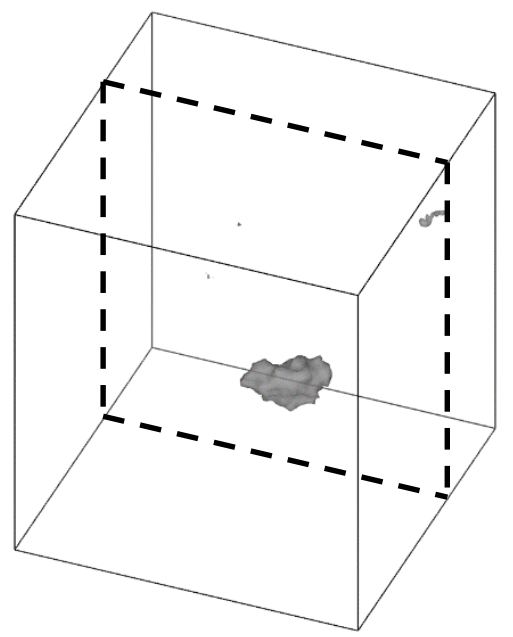

$\epsilon_{22}=0.12$

b

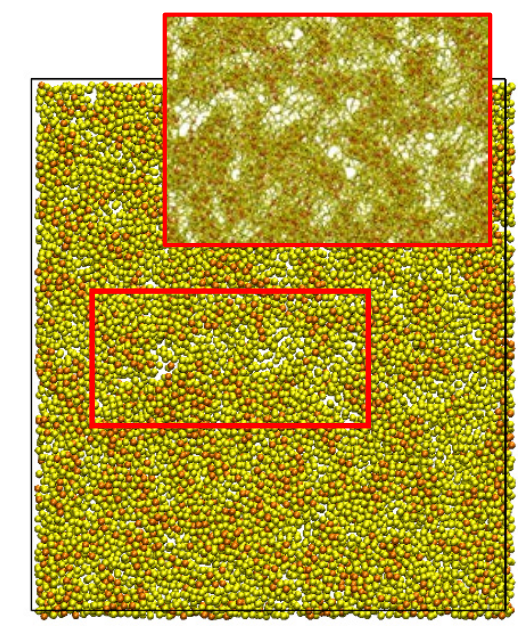

$\epsilon_{22}=0.12$

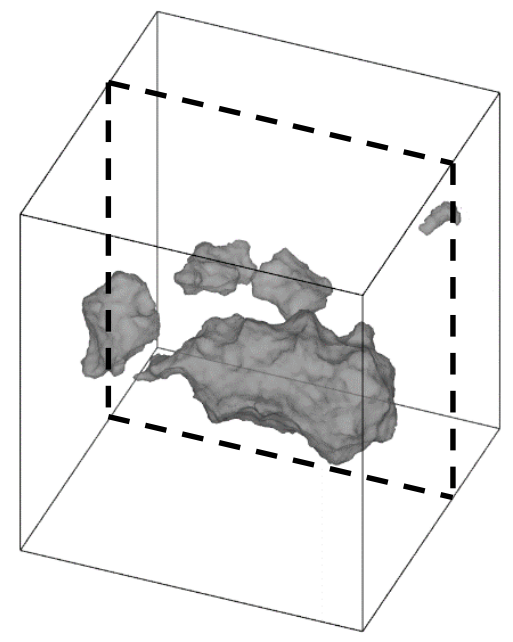

$\epsilon_{22}=0.13$

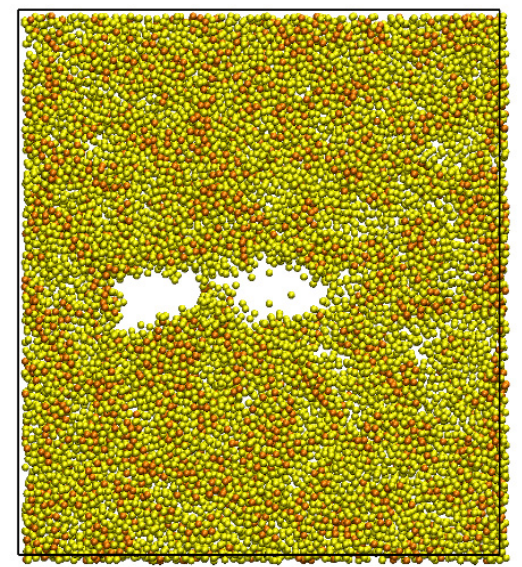

$\epsilon_{22}=0.13$

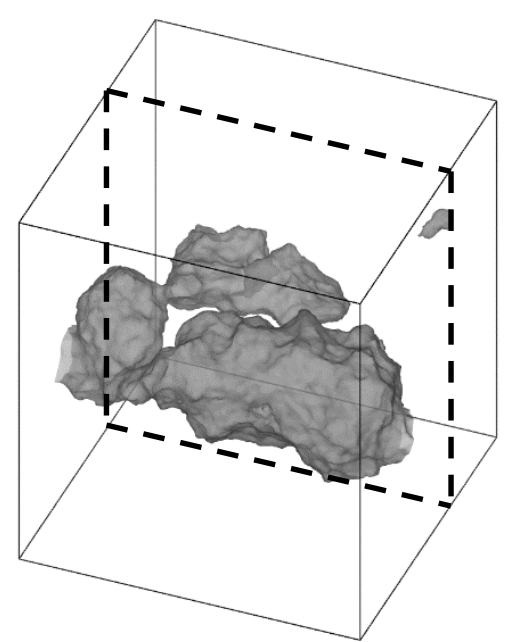

$\epsilon_{22}=0.14$

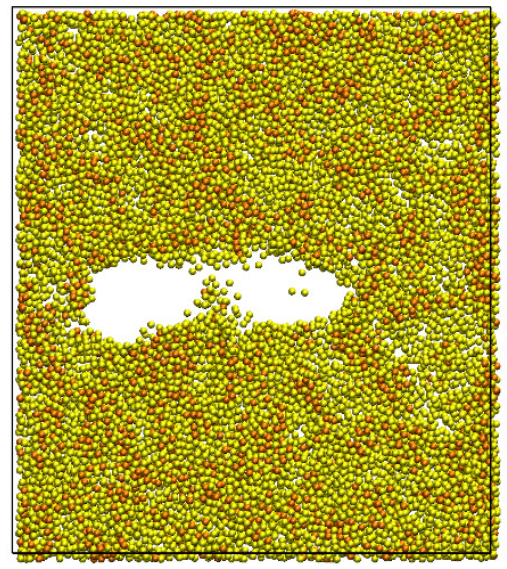

$\epsilon_{22}=0.14$

Figure 9 


$$
\epsilon_{22}=0
$$

$$
\epsilon_{22}=0.10
$$

$$
\epsilon_{22}=0.15
$$$$
\epsilon_{22}=0.20
$$

a
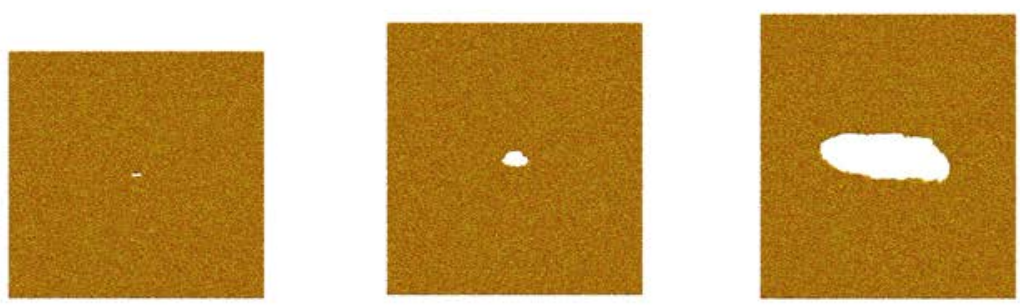

b
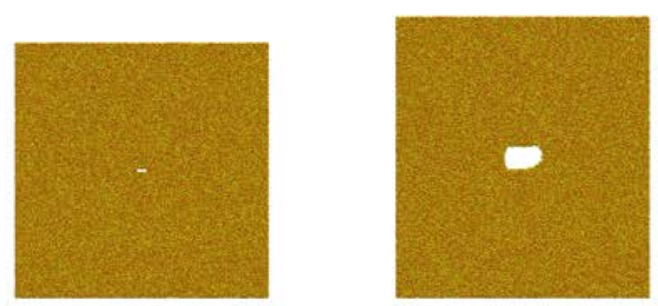

C

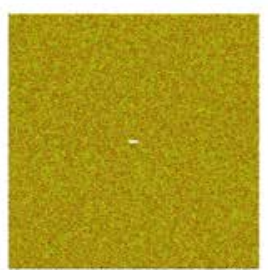

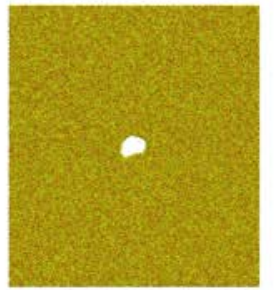
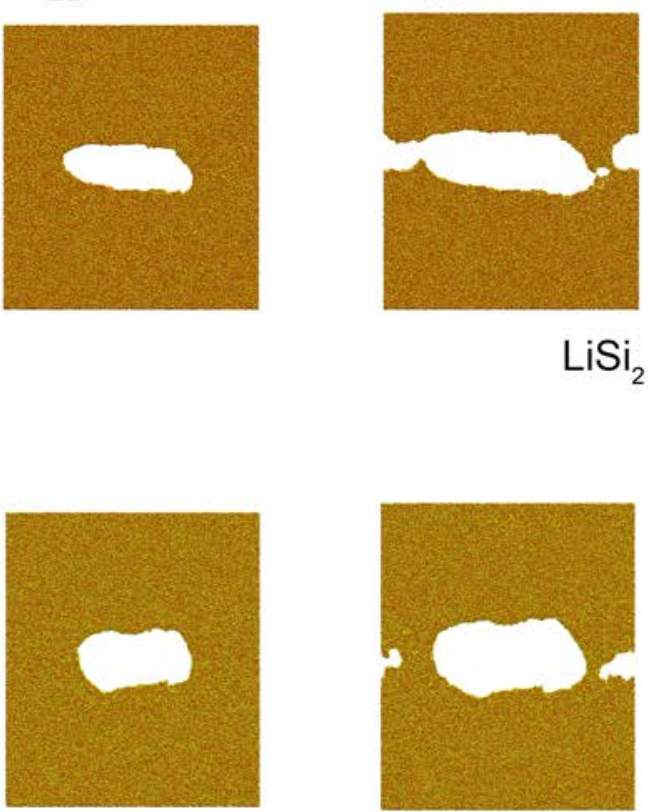

$\mathrm{LiSi}_{2}$

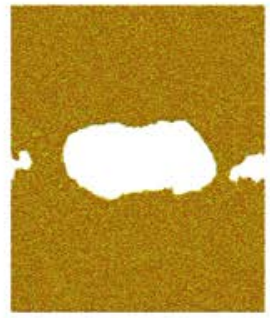

LiSi
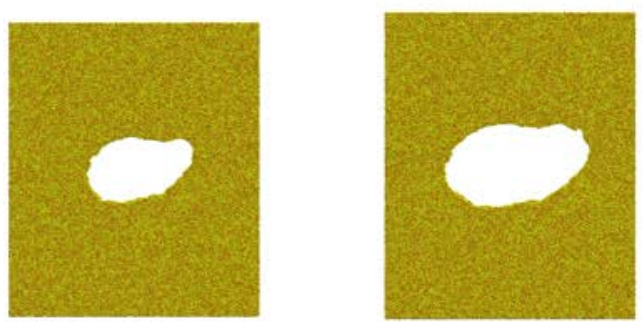

$\mathrm{Li}_{15} \mathrm{Si}_{4}$

Figure 10 